\title{
EVALUACIÓN Y OPTIMIZACIÓN DEL TRATAMIENTO TÉRMICO DE CONSERVAS DE HABAS VERDES (Vicia faba L.) EN SALMUERA
}

\section{Optimization and evaluation of the thermal treatment of canned green beans in brine}

\author{
Moisés Condori ${ }^{1}$, Antonio J Obregón ${ }^{2}$ y Américo Guevara ${ }^{2}$ \\ ${ }^{1}$ Facultad de Industrias Alimentarias, Universidad Nacional Agraria La Molina. ${ }^{2}$ Facultad de Farmacia y Bioquímica, \\ Universidad Nacional Mayor de San Marcos
}

\section{RESUMEN}

Se determinaron los parámetros de tratamiento térmico para la máxima retención del color $(5,83 \%)$ y de la textura $(2,29 \%)$ de las conservas de habas verdes (Vicia faba L.) en salmuera, a la temperatura de procesamiento de $126,7^{\circ} \mathrm{C}$ por 8,69 minutos, mediante el método de Jen et al., para lo cual se realizaron diversas pruebas con habas verdes provenientes del valle del Mantaro. Asimismo, se evaluó el tratamiento para determinar las características de penetración de calor en el punto de más lento calentamiento (a 3,8 cm de la base del envase), encontrándose los siguientes resultados: $\mathrm{f}_{\mathrm{h}}=19,34$ minutos, $\mathrm{j}_{\mathrm{h}}=$ o,46; $\mathrm{Tpsi}_{\mathrm{h}}=95,6^{\circ} \mathrm{C}, \mathrm{f}_{\mathrm{c}}=16,67$ minutos, $\mathrm{j}_{\mathrm{c}}=1,11 \mathrm{y} \mathrm{Tpsi}{ }_{\mathrm{c}}=123,2^{\circ} \mathrm{C} ; \mathrm{y}$ los tiempos de procesamiento, mediante el método de Stumbo, a las temperaturas de 104,4; 110; 115,6; 121,1; 126,7 y 132,2 ${ }^{\circ} \mathrm{C}$, encontrándose los tiempos de procesamiento 108,79; 54,76; 24,72; 13,77; 8,69 y 5,51 minutos, respectivamente, para obtener en todos los casos un mismo valor de $\mathrm{F}_{121,1^{\circ} \mathrm{C}}$ de 3,15 minutos.

Palabras clave: Habas, textura, color, sensorial, Stumbo, conservas.

\section{SUMMARY}

It was determined the parameters of thermal treatment for the maximum retention of color $(5,83 \%)$ and texture $(2,29$ \%) of canned green beans (Vicia faba L.) in brine, to processing temperature $126,7^{\circ} \mathrm{C}$ for a time of 8,69 minutes, by Jen $e t$ $a l$ method. The tests were made with green beans from Mantaro valley, in Peru. Likewise, was realized the evaluation of the thermal treatment to determinate characteristics of heat penetration in the slower warming point $(3,8 \mathrm{~cm}$ from base of can) finding the following results: $f_{h}=19,34$ minutes, $j_{h}=0,46 ; \mathrm{Tpsi}_{h}=95,6^{\circ} \mathrm{C}, \mathrm{f}_{\mathrm{c}}=16,67$ minutes, $\mathrm{j}_{\mathrm{c}}=1,11$ and $\mathrm{Tpsi}_{\mathrm{c}}=123,2^{\circ} \mathrm{C}$; and the times of processing, by Stumbo method, to temperatures 104,4; 110; 115,6; 121,1; 126,7 and 132,2 ${ }^{\circ} \mathrm{C}$, being the times of processing 108,$79 ; 54,76 ; 24,72 ; 13,77 ; 8,69$ and 5,51 minutes, respectively, to obtain in all cases the same value of $\mathrm{F}_{121,1^{\circ \mathrm{C}}}$ of 3,15 minutes.

Keywords: Green beans, texture, sensorial, Stumbo.

\section{INTRODUCCIÓN}

a especie Vicia faba L. (haba), es una de las cuatro leguminosas de grano más importantes, después del frijol, la arveja y el garbanzo ${ }^{(1)}$. El procesamiento del haba bajo la forma de conserva, es una opción para otorgarle valor agregado y expandir la actividad económica ligada a ella, logrando estabilizar su producción y sus precios.

La texturay el color son atributos muy importantes a tener en cuenta en un producto terminado, como es el caso del enlatado de habas, ya que estos, junto con el sabor y componentes nutritivos, son los más afectados durante el tratamiento térmico. En ese sentido, para elaborar conservas de habas de buena calidad es importante buscar el tratamiento térmico óptimo, de tal forma que se obtenga un producto comercial estéril microbiológicamente y que mantenga, sus atributos de calidad y componentes nutritivos.
Es conocido, que el excesivo calentamiento de los alimentos produce considerables pérdidas de nutrientes y de calidad sensorial (textura, color, sabor, etc.), por lo que se hace necesario realizar investigaciones conducentes a la obtención de parámetros óptimos de procesamiento con la menor degradación de nutrientes y factores de calidad.

Teniendo en cuenta lo antes referido, se llevó a cabo el presente trabajo de investigación, cuyo objetivo fue determinar los parámetros de tratamiento térmico óptimos para la máxima retención del color y de la textura en conservas de habas en salmuera.

\section{MATERIALES Y MÉTODOS}

\section{Materia prima}

Se utilizaron habas (Vicia faba L.), variedad Sincos, en estado de madurez pintón de color verde, 
proveniente del Valle del Mantaro, departamento de Junín.

\section{Equipos}

- Autoclave vertical, $270^{\circ} \mathrm{F}$ y $30 \mathrm{Lb} / \mathrm{plg}^{2}$.

- Sistema DATA TRACE TEMP SYSTEM, el cual está comprendido por: Un dispositivo que registra la temperatura y el tiempo, llamado TRACER MICROPACK, Un módulo PC Interface utilizado para programar y leer los tracers y Software Datatrace para Windows 95.

\section{Evaluación Sensorial y Estadística}

Se utilizó la Prueba de ordenamiento, cuyos resultados fueron tabulados en un diseño de bloque completo aleatorizado (DBCA), evaluado con la Prueba no paramétrica de Friedman, a un nivel de significancia del $5 \%{ }^{(2)}$.

\section{Metodología}

\section{Determinación del punto más frío}

Se utilizó el Sistema DATA TRACE SYSTEM, cuyos TRACER MICROPACK (dispositivos que registran la temperatura y el tiempo) fueron colocados en diferentes posiciones en el envase: en el centro geométrico, a 1/3 y a 2/3 de la base de la lata. Luego se sometió el envase a un tratamiento térmico estándar de $115,6^{\circ} \mathrm{C}$ por 30 minutos. Mediante el análisis de los datos de temperatura y tiempo, registrados por el sensor, se consideró el punto más frío el que tuvo mayor valor de $\mathrm{f}_{\mathrm{h}}{ }^{(3)}$.

\section{Determinación de los parámetros de penetración de calor}

El producto se trató térmicamente a una temperatura de $115,6^{\circ} \mathrm{C}$ por 30 minutos, registrándose los datos de tiempo-temperatura del punto de calentamiento más frío determinado anteriormente. Con los datos obtenidos, se graficaron las curvas de calentamiento y enfriamiento, a partir de las cuales se determinó: La inversa de la pendiente de la curva de calentamiento $\left(\mathrm{f}_{\mathrm{h}}\right)$; La inversa de la pendiente de la curva de enfriamiento $\left(f_{c}\right)$; La temperatura pseudoinicial de calentamiento ( $\mathrm{Tpsi}_{\mathrm{h}}$ ); La temperatura pseudoinicial de enfriamiento ( $\mathrm{Tpsi}_{\mathrm{c}}$ ); El factor de retraso en el calentamiento $\left(j_{h}\right)$ y el Factor de retraso en el enfriamiento $\left(\mathrm{j}_{\mathrm{c}}\right)$.

\section{Determinación del tiempo de tratamiento térmico} Con todos los datos de temperatura inicial de la conserva $f_{h}, j_{h}, j_{c}$, temperatura del autoclave, tiempo de levante "CUT", temperatura del agua de enfriamiento, carga microbiana inicial, probabilidad de carga microbiana final, $Z, D_{250^{\circ} \mathrm{F}}=\mathbf{0 , 2 1} \mathrm{min}$, se realizan los cálculos de los tiempos de procesamiento aplicando el método de Stumbo ${ }^{(4)}$. El valor de $\mathrm{F}_{121,1^{\circ} \mathrm{C}}$ requerido fue determinado en función a la cantidad de microorganismos iniciales en el producto antes de procesarloy al microorganismo referencial Clostridium botulinum, cuyos parámetros cinéticos son $\mathrm{D}_{121,1^{\circ} \mathrm{C}}=$ 0.21 minutos y $\mathrm{Z}=10^{\circ} \mathrm{C}{ }^{(4)}$. Posteriormente se hallaron los tiempos equivalentes necesarios para diferentes temperaturas de tratamiento térmico $(104,4 ; 110 ; 115,6$; 121,$1 ; 126,7$ y $132,2^{\circ} \mathrm{C}$ ) aplicando el método descrito por Stumbo ${ }^{(4)}$.

Determinación de la degradación de textura y color durante el tratamiento térmico

La degradación de la textura y del color se determinó mediante el método de Jen et al. ${ }^{(5)}$, en base a las combinaciones de tiempo y temperatura de procesamiento determinados por el método de Stumbo (4) y tomándose como parámetros referenciales de destrucción térmica para guisantes, cuyos valores son: $D_{121,1{ }^{\circ} \mathrm{C}}=13,2 \mathrm{~min}, \mathrm{Z}=38,33^{\circ} \mathrm{C}$, para el color, y de $D_{121,1^{\circ} \mathrm{C}}=9,2 \mathrm{~min}, \mathrm{Z}=36,67^{\circ} \mathrm{C}$, para la textura ${ }^{(6,7)}$.

\section{Optimización del tratamiento térmico}

Para este proceso se realizó una gráfica donde se relaciona el porcentaje retenido de color y textura versus los tiempos de tratamiento térmico determinados anteriormente; el punto más alto de la curva representa el óptimo, según lo recomendado por Lund ${ }^{(8)}$. Posteriormente, con el fin de validar los parámetros de optimización encontrados, se procesaron las conservas a las temperaturas estudiadas por sus respectivos tiempos de procesos de letalidad equivalente y fueron sometidas a un panel sensorial mediante una prueba de ordenamiento.

\section{RESULTADOS}

\section{Determinación del punto más frío de la conserva}

En la tabla 1 se presentan los resultados de la determinación del punto más frío, en base a los valores de $f_{h}$ de la curva de calentamiento, donde se aprecia que el mayor valor de $f_{h}$ se ubica a $1 / 3$ de la base de la lata, lo que concuerda con Ball y Olson ${ }^{(3)}$, quienes señalan que, el punto más frío de un producto en el envase, es aquel que presenta el mayor valor de $\mathrm{f}_{\mathrm{h}}$ en la curva de calentamiento. En la figura 1 también se puede apreciar que el punto, a 1/3 de la base, es el punto más frío del envase. 
Tabla 1. Valores de f para la determinación del punto más frío en conserva de habas verdes en salmuera.

\begin{tabular}{lc}
\hline \multicolumn{1}{c}{$\begin{array}{c}\text { Ubicación donde se realizó la } \\
\text { medición }\end{array}$} & $\mathbf{f}_{\mathbf{h}}(\mathbf{m i n})$ \\
\hline A 1/3 de la base (a 3,8 cm) & 19,61 \\
En el centro geométrico (a 5,6 cm) & 16,39 \\
A 2/3 de la base $($ a $7,5 \mathrm{~cm})$ & 12,55 \\
\hline
\end{tabular}

\section{Determinación de los parámetros de penetración de calor}

Las curvas de calentamiento y enfriamiento (figuras 2 y 3), obtenidas por regresión lineal, fueron utilizadas para determinar los parámetros de penetración de calor que se presentan en la tabla 2. Como se puede apreciar el comportamiento lineal de estas curvas es bastante significativo, puesto que presentan un valor de correlación lineal de 0,929 y o,9659, para el calentamiento y enfriamiento, respectivamente. Los valores de $f_{h}$ y $f_{c}$ presentan una diferencia de 2,67 minutos, que representa un $13,8 \%$ con respecto al valor de $f_{h}$.

\section{Determinación del tiempo de tratamiento térmico requerido}

Al considerar una carga inicial microbiana de 1010 UFC/g en la materia prima y estimar una reducción probable de $1 \times 10^{-12} \mathrm{UFC} / \mathrm{g}$, se obtuvo un valor de $\mathrm{F}_{121,1^{\circ} \mathrm{C}}=3,15$ minutos. Este valor es mayor al mínimo recomendado por Hersom y Hulland (13), de 2,25 minutos para conservas de $\mathrm{pH}$ mayor a 4,5 y muy cercano al indicado por la National Canners Association ${ }^{(14)}$, para guisantes en salmuera en envases de 307 x 409, cuyo valor de $\mathrm{F}_{121,1^{\circ} \mathrm{C}}$ es de 3,5 minutos.

En la Figura 4 se muestra la variación de la temperatura en el punto más frío de la conserva y del autoclave (retorta) en función al tiempo de esterilización, obtenida a partir de los datos
Tabla 2. Parámetros de penetración de calor en conservas de habas verdes en salmuera.

\begin{tabular}{cc}
\hline Parámetros del Calentamiento & $\begin{array}{c}\text { Parámetros del } \\
\text { enfriamiento }\end{array}$ \\
\hline $\mathrm{f}_{\mathrm{h}}=19,34 \mathrm{~min}$ & $\mathrm{~F}_{\mathrm{c}}=16,67 \mathrm{~min}$ \\
$\mathrm{Tpsi}_{\mathrm{h}}=95,6^{\circ} \mathrm{C}$ & $\mathrm{Tpsi}_{\mathrm{c}}=123,2^{\circ} \mathrm{C}$ \\
$\mathrm{J}_{\mathrm{h}}=0,46$ & $\mathrm{~J}_{\mathrm{c}}=1,11$ \\
$\mathrm{~T}_{\mathrm{i}}=72,5^{\circ} \mathrm{C}$ & $\mathrm{T}_{\mathrm{g}}=112,7^{\circ} \mathrm{C}$ \\
\hline
\end{tabular}

experimentales de tiempo y temperatura. Se visualiza claramente el tiempo de levante "CUT" (Come up time: tiempo necesario para que el autoclave llegue a su temperatura de trabajo), el perfil de calentamiento y enfriamiento de la conserva de habas verdes en salmuera.

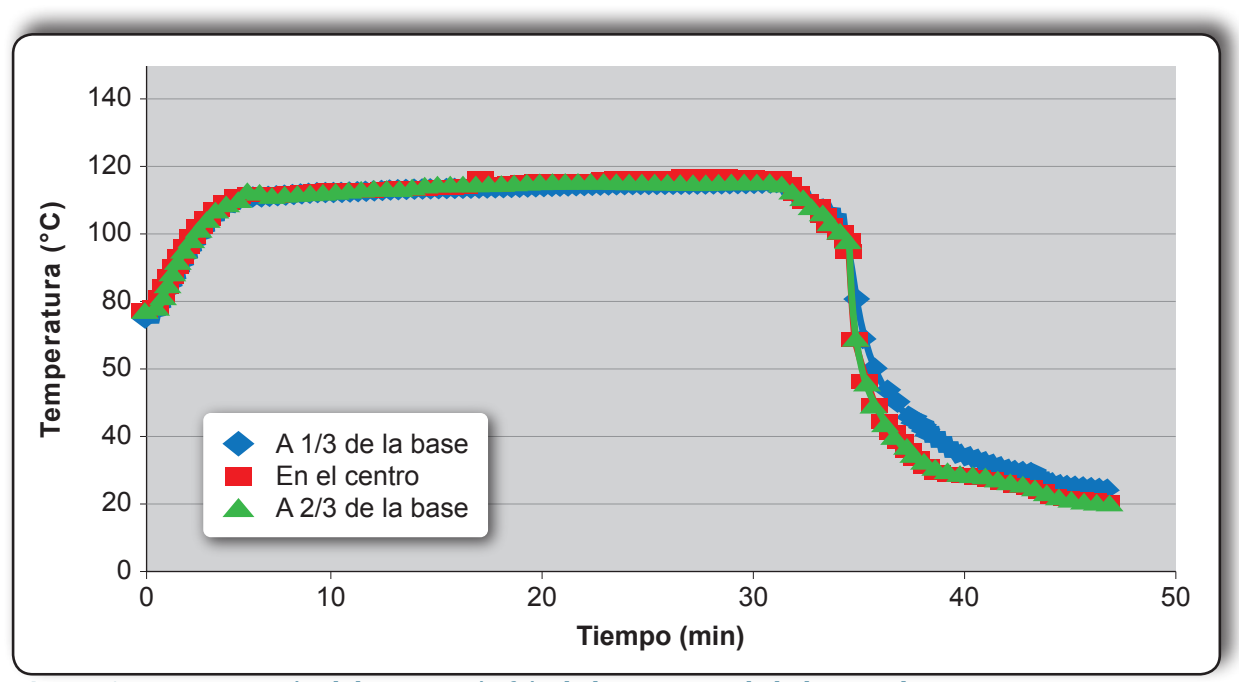

Figura 1. Determinación del punto más frío de la conserva de habas verdes.

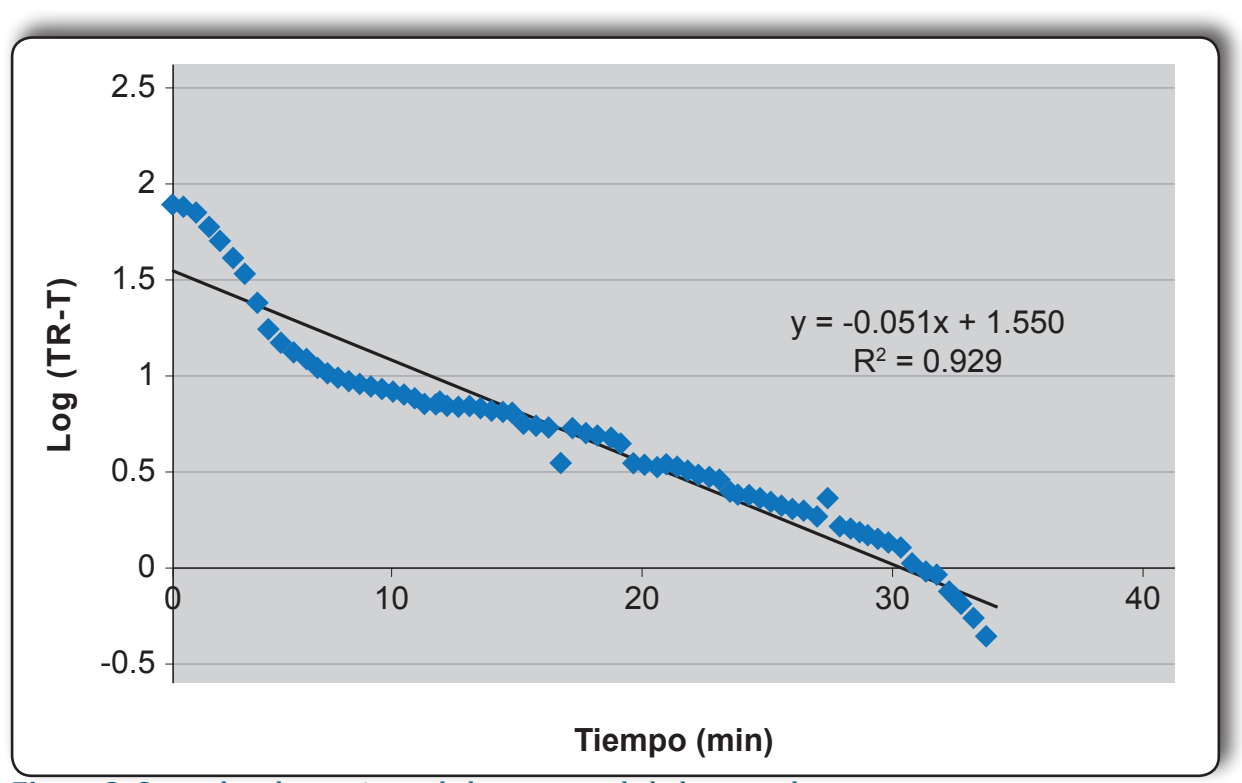

Figura 2. Curva de calentamiento de la conserva de habas en salmuera. 


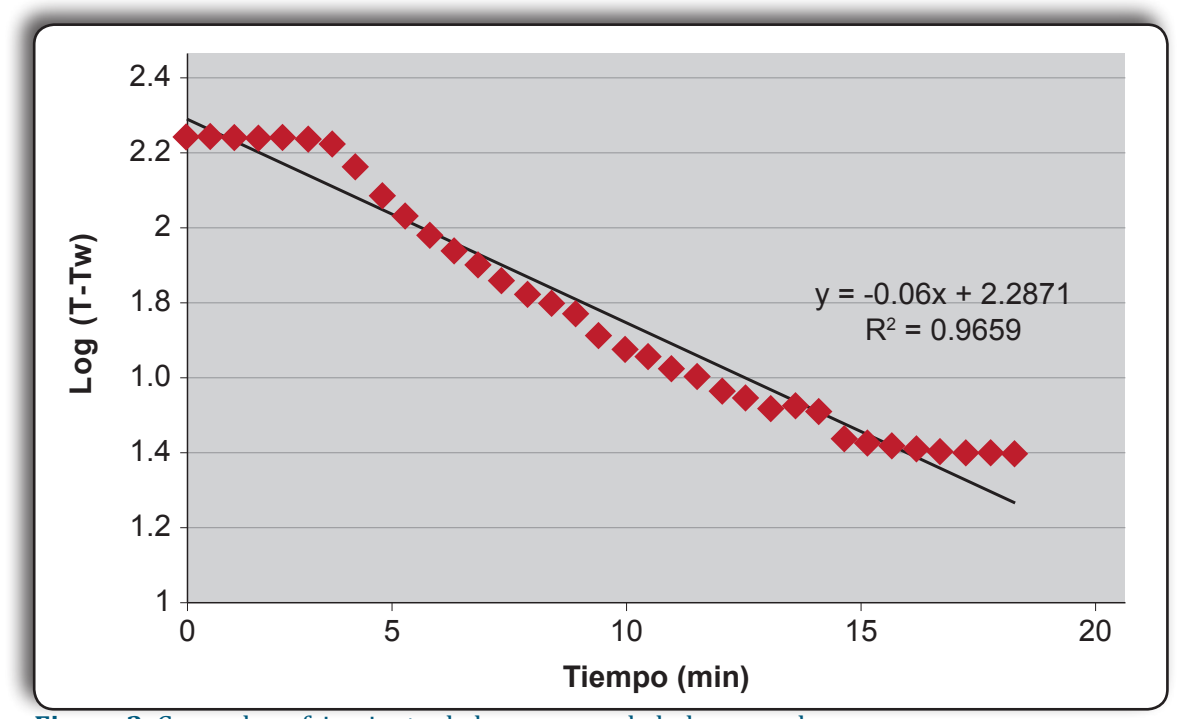

Figura 3. Curva de enfriamiento de la conserva de habas en salmuera.

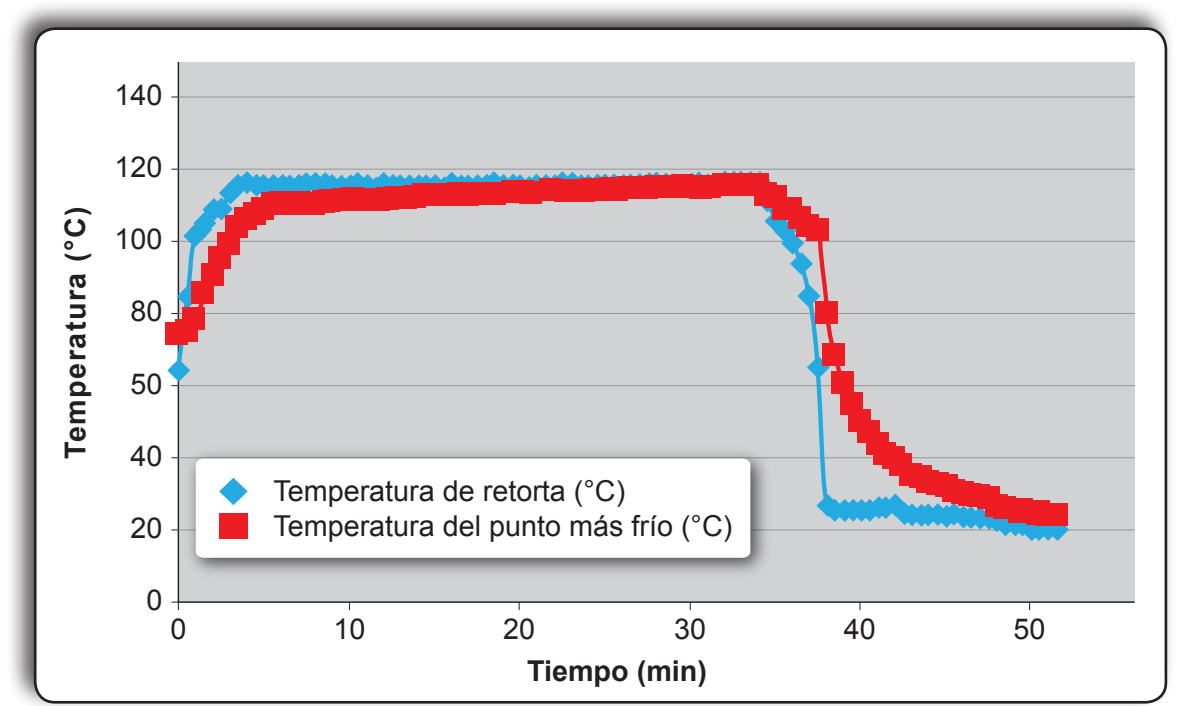

Figura 4. Curva de enfriamiento de la conserva de habas en salmuera.
54,$76 ; 24,72 ; 13,77 ; 8,69$ y 5,51 minutos, respectivamente.

Determinación de la degradación de textura y color durante el tratamiento térmico

En la tabla 3 se muestran los resultados de retención de color y textura de conservas de habas en salmuera, sometidas a diferentes temperaturas. Como se puede apreciar, a medida que la temperatura de proceso es mayor se obtienen mayores porcentajes de retención de color y de textura, por lo que los tratamientos de $126,7^{\circ} \mathrm{C}$ por 8,69 minutos y $132,2^{\circ} \mathrm{C}$ por 5,51 minutos, obtuvieron los mayores porcentajes de retención de estos factores de calidad.

\section{Optimización del tratamiento térmico}

En la figura 5 se muestra la curva de optimización del color y de la textura, en las que se aprecia la variación del porcentaje de retención del color y de la textura en función de la temperatura con su respectivo tiempo de procesamiento. Se aprecia que los factores de calidad analizados guardan una relación inversa entre el tiempo y temperatura para los procesos de optimización, es decir que a mayor temperatura se tiene una mayor retención de los factores de calidad ${ }^{(15)}$.
Con todos los datos obtenidos: temperatura inicial de la conserva $=72,5^{\circ} \mathrm{C} ; \mathrm{f}_{\mathrm{h}}=19,34 \mathrm{~min} ; \mathrm{T}_{\mathrm{r}}=121,1^{\circ} \mathrm{C}$; CUT $=3,5 \mathrm{~min}$; temperatura del agua de enfriamiento $=$ $15,6^{\circ} \mathrm{C}$; carga microbiana inicial $=1010 \mathrm{UFC} / \mathrm{g} ; \mathrm{j}_{\mathrm{h}}=0,46$, probabilidad de carga microbiana final $=1 \times 10^{-12} ; \mathrm{j}_{\mathrm{c}}=1,11$; $\mathrm{Z}=10^{\circ} \mathrm{C} ; \mathrm{D}_{121,1^{\circ} \mathrm{C}}=0,21 \mathrm{~min}$ (Clostridium botulinum), se realizan los cálculos de los tiempos de procesamiento aplicando el método de Stumbo ${ }^{(4)}$. Para dichos cálculos se consideró que, el valor de $\mathrm{F}_{\mathrm{s}}$ es igual al del $\mathrm{F}_{c}$, debido a que la conserva de habas en salmuera presenta una transferencia de calor cercana a la convección. Los tiempos de procesamiento equivalente para $F_{121,1^{\circ} \mathrm{C}}$ $=3,15$ minutos, determinados a las temperaturas de 104,$4 ; 110 ; 115,6 ; 121,1 ; 126,7$ y $132,2^{\circ} \mathrm{C}$ fueron: 108,79 ;
La validación del proceso de optimización obtenido por un panel sensorial, mediante la prueba Ranking ${ }^{(2)}$, arrojó diferencias significativas entre los tratamientos evaluados. El análisis estadístico, a un nivel de significancia del 5\% arroja diferencias significativas en la evaluación del color y de la textura, tanto para la Prueba de Friedman como para la de comparación entre tratamientos, siendo seleccionado como el mejor, el tratamiento de $126,7^{\circ} \mathrm{C}$ por 8,69 minutos.

\section{DISCUSIÓN}

De acuerdo al punto más frío de la conserva determinado, Casp y Abril ${ }^{(9)}$, señalan que, en los 
Tabla 1. Porcentaje de retención de textura y color en conservas de habas en salmuera.

\begin{tabular}{cccc}
\hline Temperatura de & Tiempo de & \multicolumn{2}{c}{$\%$ de Retención } \\
\cline { 3 - 4 } proceso $\left({ }^{\circ} \mathbf{C}\right)$ & proceso $(\mathbf{m i n})$ & Color & Textura \\
\hline 104,4 & 108,79 & 0,02 & 0,20 \\
110,0 & 54,76 & 0,04 & 0,37 \\
115,6 & 24,72 & 0,6 & 2,33 \\
121,1 & 13,77 & 1,53 & 5,28 \\
126,7 & 8,69 & 2,29 & 5,83 \\
132,2 & 5,51 & 2,81 & 6,39 \\
\hline
\end{tabular}

productos que se calientan por convección, en envases cilíndricos, el punto crítico se sitúa en el eje longitudinal a un quinto de la altura, medido desde la base, mientras que en los que se calientan por conducción, el punto crítico se localiza en el centro geométrico de su masa. Asimismo, Stumbo ${ }^{(4)}$ hace referencia que, en productos que se calientan por convección, el punto de calentamiento más frío se encuentra sobre el eje vertical, ligeramente más abajo del centro geométrico. Sin embargo, la temperatura correspondiente al centro geométrico se considera bastante aproximada al promedio efectivo del envase.

Por las referencias anteriormente expuestas y por el resultado obtenido en la presente investigación, se puede concluir que las habas enlatadas en salmuera presentan un calentamiento por convección, puesto que coincide con lo afirmado por Nickerson y Sinskey ${ }^{(10)}$ y Casp y Abril ${ }^{99}$; asimismo, el punto más frío se encuentra por debajo del centro geométrico, tal como señala Stumbo4 para este tipo de calentamientos, considerando que las habas son partículas pequeñas de alimentos suspendidas en un líquido.

En relación a los parámetros de penetración de calor, Stumbo y Longley (11) indican que el valor de $f_{h}$ igualaría exactamente el valor de $f_{c}$ en el caso de existir una convección o conducción pura, pero recomiendan que para la mayoría de los procesos debe asumirse que $f_{c}=f_{h}$ y que cuando se note una variación mayor del $20 \%$ entre ellos, debe emplearse el método general para evaluar el proceso. Por otro lado, Jen et al. ${ }^{(5)}$ señalan que $\mathrm{f}_{\mathrm{h}}$ será siempre igual a $f_{c}$, a menos que haya un cambio significativo en la difusividad térmica del producto durante el proceso, además indican que si este cambio tuviera lugar tendría que ser bastante grande, más de 10 ó 20 minutos, para influir significativamente en las relaciones $\mathrm{f}_{\mathrm{h}} / \mathrm{U}: \mathrm{g}$. De los resultados obtenidos se puede considerar que, las habas enlatadas en salmuera presentan una transferencia de calor por convección debido a que el valor de $j_{c}$ de 1,11 es cercano a la unidad, tal como lo consideró Obregón ${ }^{(12)}$ al encontrar un valor de $\mathrm{j}_{\mathrm{c}}$ de 1,4 para conservas de mandarina en almíbar.

En relación a la degradación del color de las habas verdes, Braverman ${ }^{(15)}$ indica que esta se debe a la acción de factores químicos y enzimáticos sobre las moléculas de clorofila de los vegetales. En lo que concierne a la degradación de la textura, Obregón (12), encontró resultados similares a los obtenidos en la presente investigación, al evaluar la textura de los gajos de mandarina, encontrando tratamientos que producen una mayor retención de textura al aplicar altas temperaturas por cortos tiempos.

Con respecto a la máxima retención de nutrientes, Lund ${ }^{(8)}$ refiere que en alimentos que se calientan por convección, la máxima retención de nutrientes se obtiene en procesos de alta temperatura por tiempos cortos. Asimismo, Stumbo ${ }^{(4)}$ menciona que la estabilidad térmica de la mayoría de los factores de calidad termolábiles están caracterizados por valores de $\mathrm{Z}$ muchos más altos que los que caracterizan la resistencia relativa de las bacterias. Debido a este hecho, las altas temperaturas aplicadas por tiempos cortos, que producen letalidades equivalentes a los procesos que usan temperaturas bajas por tiempos largos, son relativamente menos dañinas para la calidad de los alimentos.

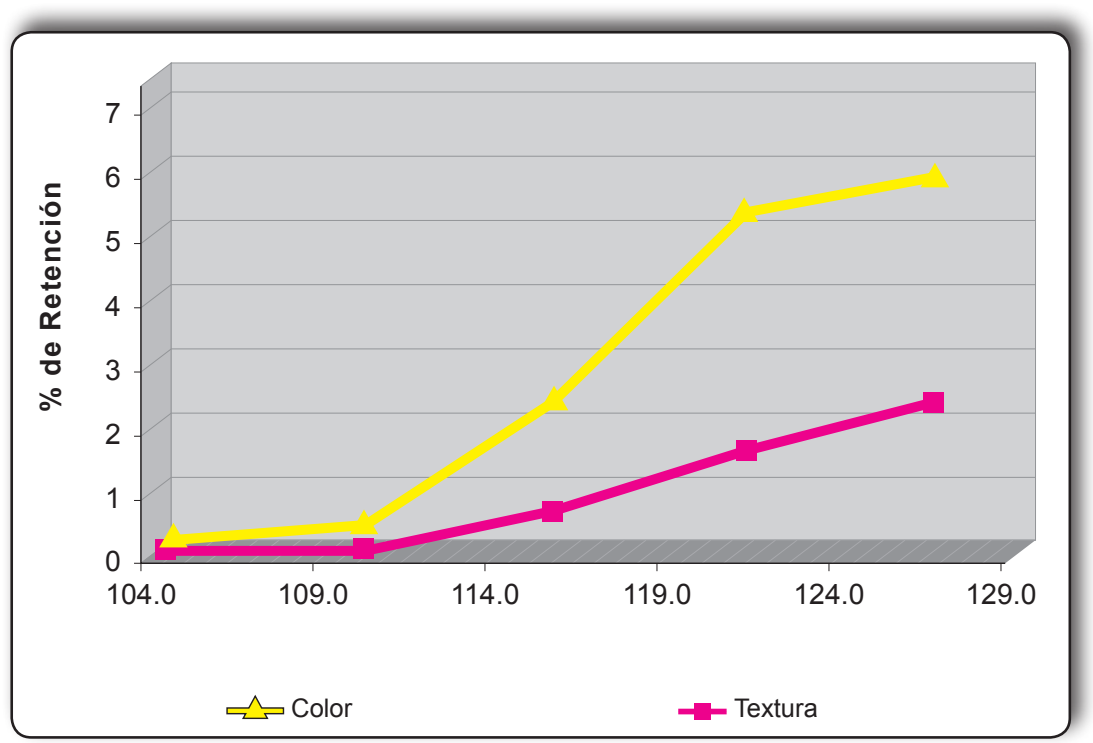

Figura 5. Curva de optimización del tratamiento térmico para la retención del color y textura en conservas de habas en salmuera. 
El tratamiento seleccionado que presentó una mayor retención de textura $(2,29 \%)$ y color $(5,83 \%)$ en las conservas de habas en salmuera corresponde a $126,7^{\circ} \mathrm{C}$ por 8,69 minutos. El tratamiento de $132,2^{\circ} \mathrm{C}$ por 5,51 minutos, a pesar de presentar una mayor retención de color y textura, fue descartado, debido a que las autoclaves de la industria alimentaria en general no están calibradas para a trabajar a estas altas temperaturas de procesamiento.

\section{CONCLUSIONES}

El punto de más lento calentamiento en la conserva de habas en salmuera se encontró a 3,8 cm de la base del envase de $300 \times 407 \mathrm{~mm}$. Los parámetros de penetración de calor encontrados para el tratamiento térmico fueron: $f_{h}=19,34$ minutos, $f_{c}=16,67$ minutos, $\mathrm{j}_{\mathrm{h}}=0,46 ; \mathrm{j}_{\mathrm{c}}=1,11 ; \mathrm{Tpsi}_{\mathrm{h}}=95,6^{\circ} \mathrm{C}, \mathrm{Tpsi}_{\mathrm{c}}=123,2^{\circ} \mathrm{C} ; \mathrm{Ti}=$ $72,5^{\circ} \mathrm{C}$ y Tg $=112,7^{\circ} \mathrm{C}$. Los tiempos de procesamiento equivalente para $\mathrm{F}_{121,1^{\circ} \mathrm{C}}$ de 3,15 minutos, determinados a las temperaturas de 104,$4 ; 110 ; 115,6 ; 121,1 ; 126,7$ y $132,2^{\circ} \mathrm{C}$ fueron: 108,$79 ; 54,76 ; 24,72 ; 13,77 ; 8,69$ y 5,51 minutos, respectivamente. El tratamiento seleccionado que presentó una mayor retención de textura $(2,29 \%)$ y color $(5,83 \%)$ en las conservas de habas en salmuera, fue de $126,7^{\circ} \mathrm{C}$ por 8,69 minutos, el cual fue validado por un panel sensorial mediante la Prueba Ranking ${ }^{(2)}$.

\section{REFERENCIAS BIBLIOGRÁFICAS}

1. Cubero Ch, Moreno M, García P. Leguminosas de grano. Mundi Prensa. Madrid, 1983.

2. Anzaldua M. La Evaluación sensorial de los alimentos teoría y práctica. Acribia. Madrid, 1994.

3. Ball C, Olson F. Sterilization in Food Technology. McGraw-Hill. New York, 1957.

4. Stumbo C. Thermobacteriology in Food Processing. Academic Press INC. USA,1973
5. Jen Y, Manson J, Stumbo C, Zahradnick J. A procedure for estimating sterilization and quality factor degradation in thermally processed foods. J Food Sci 1971; 36(4): 693-8.

6. Rao M, Lee Y, Katz J, Cooley J. A kinetic study of the loss of vitamin $C$, color and firmness during thermal processing of canned peas. J Food Sci 1981; 46(2): 636-7.

7. Rao M, Lund D. Kinetics of thermal softening of foods - A review. J Food Process Preserv 1986; 10(4): 311-29.

8. Lund D. Maximizing nutrient retention. Food Tech 1977; 31:71-8.

9. Casp A, Abril R. Procesos de conservación de alimentos. Mundi Prensa. Madrid, 1999.

10. Nickerson J, Sinskey A. Microbiología de los alimentos y sus procesos de elaboración. Acribia. Zaragoza, 1978.

11. Stumbo C, Longley R. New parameters for processes calculations. Academic Press Inc. USA, 1966.

12. Obregón A. Efecto de la temperatura sobre la textura de gajos de mandarina Satsuma (Citrus unshiu) en almíbar. Tesis de Maestría. Universidad Nacional Agraria La Molina; Lima, 2001.

13. Hersom, A, Hulland E. Conservas Alimenticias, Procesado Térmico y Microbiológico. Acribia. Zaragoza,1984.

14. National Canners Association Research Laboratories: Laboratory Manual for Food Canners and Processors. The AVI Publishing Company, Inc. Connecticut,1968.

15. Toledo T. Fundamental of food process engineering. Aspen Publishers, Inc. Maryland, 1999.

Manuscrito recibido el: 05/02/2013

Aceptado para su publicación el: 01/04/2013

\section{Correspondencia:}

Nombre: Antonio José Obregón La Rosa

Dirección: Jr. Puno 1002 Lima 1-Perú

Email: antoniojose_obregon@yahoo.com 